DOI: https://doi.org/10.47133/38209

BIBLID: 0251-2483 (2020-2), 255-286

\title{
LA MEMORIA DE LA GUERRA DE LA TRIPLE ALIANZA EN LA ESCUELA PARAGUAYA (1989-2020): NACIONALISMOS, IDENTIDADES Y ENSEÑANZA DE LA HISTORIA
}

\section{THE MEMORY OF THE WAR OF THE TRIPLE ALLIANCE}

IN THE PARAGUAYAN SCHOOL (1989-2020): NATIONALISMS, IDENTITIES AND HISTORY TEACHING

\section{Carolina Alegre Benítez ${ }^{1}$}

Enviado: $27 / 07 / 2020$

Aceptado: 10/12/2020

Resumen: El estudio se propone explorar la representación escolar de la Guerra de la Triple Alianza presente en un conjunto de manuales escolares de Historia y Geografía y Ciencias Sociales empleados en Paraguay durante el período 1989-2020, con un doble objetivo: primero, poner de manifiesto las tensiones provocadas entre los objetivos románticos (con fines identitarios) e ilustrados (con fines críticos) en la enseñanza de la historia; segundo, analizar las formas en que las representaciones hegemónicas de la guerra operan como un elemento configurador de la identidad nacional. En una sociedad cada vez más global que afecta las relaciones decimonónicas entre enseñanza de la historia y construcción de la identidad nacional, el modelo de historia escolar en clave romántica y ligado a las emociones parece proyectarse en Paraguay hasta la actualidad.

Universidad de Granada, España. Correo de contacto: carolinalegre@ gmail.com 
Palabras clave: Paraguay; Guerra de la Triple Alianza; identidad nacional; manuales escolares; enseñanza de la historia.

Abstract: The study aims to explore the school-based representation of the War of the Triple Alliance present in a set of History and Geography and Social Science school textbooks used in Paraguay during the period 1989-2020, with a twofold objective: first, to highlight the tensions caused between romantic objectives (for identity purposes) and enlightened ones (for critical purposes) in the teaching of history; second, to analyze the ways in which hegemonic representations of war operate as a shaping element of national identity. In an increasingly global society that affects the nineteenth-century relations between history teaching and the construction of national identity, the model of school history in a romantic key and linked to emotions seems to be projected in Paraguay until today. Keywords: Paraguay; War of the Triple Alliance; national identity; school textbooks; history teaching. 


\section{Introducción}

Las sociedades contemporáneas recuerdan sus guerras a través de diversos dispositivos entre los que cabe citar las prácticas sociales y producciones culturales como publicaciones diversas, cancioneros, cine, monumentos y manuales escolares. (Capdevila, 2006). Las instituciones escolares constituyen escenarios privilegiados para la conformación de la memoria colectiva, allí se transmiten conocimientos específicos, valores, reglas sociales y sentimientos de pertenencia nacional; en este marco cobra protagonismo la historia escolar, configurada a finales del siglo XIX como una asignatura destinada a la formación de ciudadanos nacionales (Jelin y Lorenz, 2004).

Sin embargo, la actual coyuntura global afecta de forma profunda las relaciones entre estado, escuela e historia articuladas hace ya más de un siglo. Las contradicciones entre una enseñanza de la historia con fines identitarios y una enseñanza de la historia con fines de racionalidad crítica resultan frecuentes. En la configuración de las memorias colectivas, la historiografía y la historia escolar constituyen puntos de apoyo, no exclusivos, del campo donde se juega el recuerdo del pasado. En este complejo escenario: ¿qué se espera de la escuela? Y en relación con la enseñanza de la historia ¿cómo abordar con los estudiantes el pasado conflictivo?, ¿cómo decidir cuando las controversias sobre el pasado están presentes en la vida política y cotidiana de una sociedad? (Carretero, 2007, Cuesta, 2011).

En la construcción de la memoria histórica en la escuela es posible distinguir tres registros del pasado: la historia académica, que desarrollan historiadores y científicos sociales; la historia escolar, que se enseña en las escuelas y tiene fuertes vínculos con las emociones; y la historia cotidiana, que forma parte de la memoria colectiva del conjunto de la sociedad. Estos tres registros de la historia no se encuentran aislados sino que forman parte de una estructura de narraciones que establecen relaciones entre sí y que intervienen en diferentes dominios de la subjetividad, se trata de tres espacios de la historia que se corresponden con tres formas de construcción social y 
significativa del pasado y que intervienen en la configuración de la identidad colectiva de cada individuo (Carretero, 2007).

Teniendo en cuenta las cuestiones planteadas hasta aquí, el artículo se propone explorar las dificultades que implica abordar la representación escolar de uno de los acontecimientos históricos constitutivos de la identidad nacional paraguaya: la Guerra de la Triple Alianza. A finales del siglo XIX y comienzos del siglo $X X$ se conforma una memoria colectiva fuertemente unida a una representación viva del Paraguay como una nación gloriosa y donde la Guerra de la Triple Alianza se convierte en un acontecimiento articulador del pasado nacional. Frente al relato de los países vencedores, surgió un discurso profundamente nacionalista que contribuyó a perpetuar una imagen estereotipada de los cuatro países contendientes y de los actores sociales que participaron en la guerra (Brezzo, 2008; Capdevila, 2009; Soler, 2010).

La propuesta se organiza en dos objetivos: a) explorar los relatos escolares acerca de la guerra presentes en una selección de manuales escolares de historia empleados entre 19892020, poniendo de manifiesto las tensiones provocadas entre los objetivos románticos (con fines identitarios) e ilustrados (con fines críticos) en la enseñanza de la historia paraguaya; b) analizar las formas en que las representaciones hegemónicas de la guerra operaron como un elemento configurador de la identidad nacional, afianzando un modelo de historia escolar en clave romántica que parece proyectarse hasta la actualidad.

Todo ello, teniendo en cuenta el contexto de una escuela y sociedad cada vez más global, donde la versión "oficial" o estatal de la historia se ve cada vez más confrontada con otras narrativas históricas, alternativas o incluso contradictorias, sobre la Guerra de la Triple Alianza. La metodología en la cual se enmarca el estudio corresponde a los métodos cualitativos de las Ciencias Sociales y para el análisis se combinan técnicas provenientes del análisis de contenido y del análisis del discurso. De forma específica, el recorrido atiende a dos variables: causas de la 
guerra y construcción de la imagen de "nosotros"/"Ios otros".

\section{Nacionalismo, identidad y memoria en Paraguay}

Después de la guerra, una élite muy próxima a la ideología liberal en los aspectos político y económico, aunque conservadora en lo social, asume la reorganización del país bajo la consigna de la "regeneración", en estos años la educación en general y la enseñanza de la historia en particular desempeñarían una función crucial en la legimitación de un discurso histórico acerca de la historia y de la guerra (Velázquez, 2015). Hacia 1880 la historia nacional se incluiría como asignatura obligatoria desde el primer grado, para la enseñanza de la historia en las escuelas se adoptó el modelo historiográfico impulsado por Cecilio Báez, intelectual paraguayo adscrito a una corriente historiográfica que articuló una lectura de la historia nacional en la que se consideraba al presidente Francisco Solano López como responsable de la Guerra de la Triple Alianza. En realidad, existía un discurso escolar que se pretendía hegemónico y que entraba en conflicto con otros relatos posibles de la guerra que circu- laban en los espacios educativos (Brezzo, 2001; Telesca, 2015).

En las primeras décadas del siglo XX el proyecto de reconstrucción nacional planteado en términos de regeneración se había desdibujado y frente al relato de la guerra ajustado a la visión de los vencedores se va conformando una "contramemoria", surge una corriente historiográfica nacionalista que producirá un discurso de la guerra basado en el heroísmo y el sacrificio del pueblo paraguayo y presentará al Paraguay como una gloriosa nación (Brezzo, 2008; Capdevila, 2009). Más tarde, en plena efervescencia del sentimiento nacional por la victoria de la Guerra del Chaco, el nuevo régimen instaurado a partir del año 1936 instalará el nacionalismo como política de estado y asimismo se ocupará de suscitar un orgullo nacional especialmente herido por la derrota sufrida en la guerra, el discurso patriótico nacionalista se consolidará a partir de 1954 con el inicio 
de la dictadura de Stroessner (Brezzo, 2009; Capdevila, 2009).

De acuerdo con Nickson (2010) el régimen de Stroessner empleo hábilmente el discurso de la identidad nacional con el objetivo de reforzar su legitimidad, adoptó la “contra-narrativa" de carácter patriótico y dispuso la historia al servicio de sus intereses. El sistema público de educación, a través de asignaturas como Historia y geografía e Instrucción Cívica, así como la literatura y la prensa, se convirtieron en canales propicios para la difusión del ideario del régimen. En la narrativa nacionalista Stroessner representaba la continuación de la línea histórica trazada por los héroes de la patria, de tal manera que aparecía como el sucesor natural de Francisco Solano López. Al mismo tiempo, el régimen fue hábil en aprovechar para sus intereses los sentimientos populares contra la "invasión extranjera" que se remontaban a la época de guerra.

Con la instauración de la dictadura de Stroessner en el año 1954, se producen una serie de cambios en las prácticas escolares y en la ingeniería normativa educativa como parte de la transformación radical que llevó adelante el orden político autoritario (Soler, Elías y Portillo, 2015). En relación con la enseñanza de la historia, durante este período la denominada pedagogía nacionalista ocupó un lugar predominante en las escuelas, cristalizado en la política educativa, en la normativa y en el currículo; se adopta la modalidad de "libro único" y se encomienda la redacción de todos los contenidos de historia al profesor Luis Guillermo Benítez (Brezzo, 2001; 2009).

Elías y Segovia (2011) mencionan una serie de elementos simbólicos del stronismo que circulaban en las escuelas y que transmitían un modelo de organización acorde con el ideario del régimen. Entre estos conceptos y mecanismos se encuentra la mistificación de los héroes, que se refiere a la construcción de un relato del pasado nacional organizado en torno a las figuras de algunos héroes a los que se atribuía aptitudes extraordinarias y a la enseñanza de una historia lineal que omitía ciertos períodos de la historia como el de los gobiernos liberales y febre- 
rista. De igual manera, Velázquez (2018) señala que durante el gobierno de Stroessner en los manuales escolares la historia se presentaba de manera teleológica, con marcado empleo de verbos impersonales que reforzaban una presentación naturalizada de los hechos del pasado; en definitiva un relato escolar donde Stroessner vendría a completar una línea de grandes gobernantes en una historia paraguaya épica y heroica.

Destaca Barreto (2013) que el discurso heroico de la guerra se cristalizó en los manuales escolares empleados en las escuelas durante toda la segunda mitad del siglo XX, y entre los personajes incluidos en el relato escolar destacaría la figura de las residentas como aquellas mujeres que siguieron incondicionalmente a López hasta el aciago final; en el otro extremo estarían aquellas mujeres que no lo hicieron y que fueron aniquiladas por traidoras. Salvo algunas excepciones, la historiografía oficial nacionalista organizada a comienzos del siglo XX y reproducida en las décadas siguientes rescató la figura de tres personajes femeninos asociados a la guerra: en primer lugar las residentas, que representaban el modelo femenino ideal, y de forma secundaria las destinadas y las reconstructoras (Alegre, 2016).

\section{Transición democrática y educación: cambios y persis- tencias}

Acerca del proceso de transición que se inicia en el año 1989 Abente (2010) plantea que el fin de la dictadura significó sobre todo el desmantelamiento del sistema autoritario de dominación política, donde el estado se identificaba al mismo tiempo con el Partido Colorado y con las Fuerzas Armadas; destaca que el triunfo del golpe adquirió desde el primer momento un matiz discordante porque al tiempo que se asumía que el golpe de estado enarbolaba la bandera de la democracia, también se argumentaba la necesidad del golpe como una forma de asegurar la continuidad del Partido Colorado en el gobierno.

Desde el inicio de la transición se proyectó la transformación del sistema educativo, una propuesta que implicó la revisión de la pedagogía nacionalista, 
Rivarola (2000) recuerda que a pesar de las fuertes críticas extendidas hacia el aparato educativo de la dictadura, la vigilancia y tutela que ejerció el nuevo gobierno no produjo cambios sustanciales en la educación. Asimismo, D'Alessandro (2014) advierte que los promotores del proceso de institucionalización democrática fueron los mismos actores políticos que, en mayor o en menor medida, ocuparon puestos de poder durante los treinta y cinco años de gobierno autoritario.

En cualquier caso, al comienzo de la transición se dispuso la revisión del conjunto de la política educativa paraguaya, así como del currículo escolar y de los planes de estudio, un esfuerzo del conjunto de actores políticos que acabó por confluir en el proceso de Reforma Educativa iniciado a partir del año 1992. Resulta importante destacar que en el marco de las iniciativas de renovación del sistema educativo se llevó a cabo un estudio de los manuales de Historia y Ciencias Sociales empleados en las décadas anteriores. Los resultados de este estudio fueron presentados en un documento elaborado por la Academia Paraguaya de la Historia, las propuestas de renovación para la enseñanza de la historia que se desprendían del mismo fueron aprobadas por el Consejo de la Reforma Educativa durante la presidencia de Wasmosy (Brezzo, 2001; Rivarola, 1996).

El documento "Propuestas de la Academia Paraguaya de la Historia para la reforma de programas y textos escolares de la materia" (Historia Paraguaya, 1996) fue elaborado por una comisión integrada por las profesoras Margarita Durán Estragó, Milda Rivarola y Margarita Prieto Yegros. La finalidad principal de este estudio fue: "[...] estudiar los manuales de Historia y Ciencias Sociales empleados en las últimas décadas en las distintas escuelas y colegios del país, hacer una evaluación crítica de los mismos y diseñar propuestas de reforma relativas a metodología, principios y contenidos de los currículums y textos de historia paraguaya" (p. 255).

De forma muy resumida, el estudio crítico planteaba que el carácter belicista de la enseñanza de la historia necesitaba ser revi- 
sado, un enfoque que resultaba especialmente evidente en los capítulos dedicados a la Guerra de la Triple Alianza y la Guerra del Chaco, que ocupaban una extensión desmesurada respecto al conjunto de contenidos de los manuales. Además, el estudio ponía de relieve que no se registraba visión crítica alguna en los manuales escolares, que presentaban: “[...] la historia mítica del héroe máximo de la nacionalidad y del heroísmo incomparable de soldados, mujeres y niños" (p. 264). Entre las propuestas se mencionaba la urgencia de dejar de presentar la historia escolar de los manuales como "parábola moralizante" y en cambio considerarla en su carácter científico. Y en relación con la preferencia que otorgan los manuales a la historia gubernamental y a las dos guerras con sus respectivos héroes, se insta a relativizarlas en el conjunto de procesos históricos más amplios.

Hace más de dos décadas que se inició el proceso de transformación educativa, durante este tiempo el país ha atravesado diferentes gobiernos, crisis y coyunturas que deben ser consideradas en el contexto de los cambios producidos a nivel regional y global. Numerosos informes y estudios, desde diversos enfoques y disciplinas, han puesto en perspectiva las transformaciones del sistema educativo experimentadas a partir de la transición en un complejo contexto que siempre arroja luces y sombras. Pero ya a comienzos de la década de 2.000, el Informe del Consejo Nacional de Educación y Cultura sobre la situación de la educación en el Paraguay (Consejo Nacional de Educación y Cultura, 2002: p. 19) hacía un recuento de los 12 años de gobierno democrático y se pronunciaba a modo de presagio: "La llamada "transición a la democracia" está estancada desde hace años. Las nostalgias de algunos ciudadanos y políticos que se anclaron en criterios y gustos de gobierno y gestión propio de las dictaduras, siguen teniendo peso y presencia en el ámbito de la educación".

Más reciente, el estudio de Elías (2014) sobre la Reforma Educativa sostiene que el cambio político iniciado en 2008 con la llegada a la presidencia de Fernando Lugo aportó nuevas ideas a la formulación de políticas sociales en consonancia 
con las políticas educativas impulsadas en la región. Sin embargo, estos nuevos planteamientos e ideales se plasmaron en algunas acciones concretas pero no lograron extenderse al conjunto de la política educativa.

En el plano los discursos historiográficos acerca de la guerra, Brezzo (2009, 2010) afirma que a pesar de las limitaciones del desarrollo de la historia en Paraguay es posible observar la emergencia de nuevos enfoques y perspectivas de estudio en un esfuerzo por superar las miradas nacionales y advierte que el debate intelectual en torno al conflicto se redoblaba multiplicando las controversias. Con otro matiz, Lambert (2016) afirma que en el contexto de la caída del régimen y el inicio de la transición la literatura contestataria de las versiones oficiales de la historia aumentaron de forma notable, sin embargo, en general la transición política y el restablecimiento gradual de las libertades civiles y políticas no suscitaron ningún reto, revisión o rechazo significativo del discurso nacionalista.

Para Capdevila (2009: párr. 29), desde Franco hasta
Stroessner los regímenes militares instauraron una historia oficial nacionalista y desplegaron una política conmemorativa, dos elementos que han modelado el "macizo de la Guerra de la Triple Alianza como substrato de la identidad nacional", por esta razón el autor plantea la necesidad de revisar la historia y la relación que la sociedad establece con el pasado. Por un lado, admite que han florecido nuevos discursos historiográficos e interés por parte de la ciudadanía, los sucesivos gobiernos dejaron de imponer una visión hegemónica del pasado nacional y los nuevos debates encontraron eco en la escuela con la publicación de renovados manuales escolares, una coyuntura que reblandece el "macizo de la guerra"; por otro lado, señala que no se produjo una ruptura clara entre dictadura y democracia y el discurso nacionalista conformado en torno a las políticas identitarias de comienzos del siglo XX no ha desaparecido.

Velázquez (2011; 2018) pone en cuestión el nacionalismo heroico tradicional que inventó y legitimó un pasado nacional sin fisuras y advierte 
de la necesidad de "reparar el pasado", en el sentido de desplegar prácticas historiográficas que proyecten un Paraguay capaz de incluir distintas memorias, con sus tensiones y conflictos inherentes. Hace notar el agotamiento del discurso heroico en la sociedad y observa que en los programas de estudio recientes se aprecian cambios hacia una visión más amplia de los procesos históricos que incluyen abordajes interdisciplinarios; la escuela propone un ejercicio de la ciudadanía que implica la participación activa y democrática de los estudiantes.

En relación con los personajes vinculados al relato patriótico de la guerra, Potthast (2006) plantea que hasta la caída del régimen de Stroessner la figura de la residenta fue siempre la personificación de los valores nacionales, si bien con el tiempo se han rescatado la figura de las destinadas y otras formas de participación de las mujeres en la contienda. Por otra parte, recuerda que en las instituciones educativas ya no se aprende la obra poética "La mujer paraguaya" (1899) que sintetiza el rol tradicional que los nacionalistas asignaban a las mujeres; ahora se lee en las aulas novelas como "Pancha" (2000), de la autora Maybell Lebron. Merece la pena recordar que Pancha Garmendia fue una mujer destacada de la sociedad de su época a la que Francisco Solano López cortejó por largo tiempo sin éxito.

\section{La memoria de la Guerra de la Triple Alianza en los ma- nuales escolares}

\subsection{Antecedentes y causas del conflicto}

El manual escolar Estudios Sociales 2 Curso, de la profesora Irmina Claude de Lezcano, está dirigido al estudiantado de $2^{\circ}$ curso del nivel secundario del antiguo sistema educativo. Editado por Talleres Gráficos Comuneros, no cuenta con una fecha de edición visible, como suele ocurrir con algunos manuales editados en la década de los ochenta. En las primeras páginas se advierte: “Estudios Sociales, 2er. curso básico. Reajustado al programa del Ministerio de Educación y 
Culto", datos que indican que se trata de un libro editado con posterioridad al año 1975.

Hay que tener presente que los manuales escolares de Talleres Gráficos Comuneros fueron empleados tanto en escuelas públicas como privadas durante toda la década de 1990. Además, los materiales educativos de la profesora Irmina $C$. de Lezcano tienen un espacio muy importante en el Programa de Estudios Sociales reimpreso 1990, de hecho, junto con los libros de Luis G. Benítez, son los más nombrados en los apartados de "Bibliografía recomendada".

En el manual Estudios Sociales 2 el contenido de la guerra ocupa dos capítulos extendidos en 10 páginas, sin embargo los antecedentes y causas de guerra aparecen en el capítulo anterior bajo el epígrafe "Situación del país y del Río de la Plata en dicha época". Lo primero que hay que hacer notar en este apartado es la estabilidad y prosperidad que se atribuye al período inmediatamente anterior al inicio del conflicto: "El gobierno de Don C. Antonio López fue muy fructífero en realizaciones, es así como organizó el Estado y sus instituciones" (Lezcano, s/f.: p. 244). El relato no introduce apreciaciones acerca del heroísmo del pueblo paraguayo, aunque sí menciona el valor demostrado por dos personajes masculinos en el apartado de "Campañas militares", las actuaciones de Bernardino Caballero y el coronel Francisco Martínez.

El manual escolar Paraguay Ñane Retã Geografía. Historia. Educación Cívica (ñane retã significa en lengua guaraní: nuestro país), editado en 1998 por la editorial En Alianza (y reeditado varias veces sin variaciones en la década siguiente), está realizado por un equipo de autores bajo la dirección de Carmen Cosp de Santa Cruz y la coordinación de María Teresa Ferreira Escobary el responsable de la edición es Pedro Saccaggio. La publicación de este manual marcó el paso de un único autor a un equipo de autores, además en este caso los autores intentaron incorporar los avances de la historiografía paraguaya (D'Alessandro, 2014). Es uno de los primeros libros de texto editados conforme a los nuevos Programas de Estudio elaborados para el Tercer Ciclo de la Educación Escolar 
Básica. Tanto en el formato como en el contenido introduce novedades significativas.

En el manual Paraguay Ñane Retã el contenido de la guerra se extiende en dos capítulos de seis páginas cada uno. Merece la pena tener en cuenta que este manual escolar dedica el mismo número de páginas al tratamiento de la guerra y al abordaje del período de postguerra. A lo anterior hay que agregar que gran parte de los capítulos que desarrollan los contenidos del área de Historia tienen también 6 páginas de extensión. Por lo tanto, y a diferencia de otros manuales escolares ya analizados, en el manual Paraguay Ñane Retã no se le otorga al tema de la guerra contra la Triple Alianza un espacio central en el conjunto del libro.

En rigor, el inicio del Capítulo 21 "La guerra contra la Triple Alianza" no hace referencias al pasado heroico y glorioso del Paraguay en los años anteriores al comienzo de la guerra de contra la Triple Alianza, pero conviene apuntar algunas cuestiones interesantes del comienzo del relato. En el primer apartado del capítulo, “Francisco Solano
López sucede a su padre", es posible identificar un cambio de registro respecto del relato que ofrece la historiografía oficial paraguaya. De hecho, ya en el último apartado del capítulo anterior "La gestión de Carlos A. López", se señalan las obras y avances llevados a cabo durante su gobierno, pero sin designar este período como "la edad de oro" de la historia paraguaya; el tono empleado aquí es distinto, cuando se afirma: “La gestión de López trajo cierto bienestar para los paraguayos" (Saccaggio et al., 1998: p. 128).

En relación con los antecedentes, el relato resulta similar a otros manuales escolares ya analizados, pero la explicación de la sucesión en el gobierno y la elección de Francisco Solano López introduce nuevos elementos: "Una vez reunido el Congreso, el diputado Varela junto con otros, trataron de evitar la elección del general, alegando que el gobierno de la República no era patrimonio de una familia. Sin embargo, el intento fue acallado y Solano López fue elegido presidente por un período de 10 años" (p. 131). 
El apartado que aborda las causas del conflicto bélico se presenta con un título significativo que adelanta el contenido del mismo: "Las causas de la guerra: un tema polémico". Antes de plantear de forma sucinta las causas, se aclara que es un tema muy discutido por los historiadores y que existen diferentes posturas acerca del tema. Las causas de la guerra presentadas en este manual intentan reflejar diferentes corrientes historiográficas, aunque el relato no mencione de forma explícita dichas corrientes.

La primera causa pone el acento en el peligro que suponen para el equilibrio del Plata las acciones del Brasil: "La versión oficial de la época, sostiene que el Paraguay defendía la libre navegación de los ríos, y la ocupación del Uruguay por el imperio brasileño suponía riesgos para el equilibrio del Plata" (p. 131). En la segunda causa emerge un nuevo actor: "Los intereses económicos de Inglaterra que pretendía someter el modelo económico autosostenible del Paraguay y lograr la apertura a sus productos" ( $p$. 131). Como novedad, la tercera causa mencionada se aleja del discurso oficial incluyendo entre los responsables del inicio del conflicto bélico al Paraguay y a López: "La agresión militar de parte de Paraguay (Matto Grosso y Corrientes). El despotismo y las ambiciones políticas de López" (p. 131). La cuarta y última causa hace referencia a múltiples factores: "La de los historiadores contemporáneos, que incluye varios elementos: persistencia del problema indefinido de fronteras, impericia de la cancillería paraguaya, imposición de la hegemonía regional por Brasil y Argentina, etc." (p. 131).

El manual escolar Historia y Geografía 8 Grado editado en el año 2000 por la editorial Don Bosco es el resultado de una licitación realizada por autoridades competentes del Ministerio de Educación y Cultura a finales de la década de 1990. Constituye un excelente ejemplo de los esfuerzos Ilevados a cabo por el MEC en la primera etapa de la implementación de la Reforma Educativa en el Tercer Ciclo de la EEB. La redacción del texto y contenido corresponde a María Graciela Monte de López Moreira, Ana Carolina Reyes Romero y Juana Dávalos (para contenidos en 
lengua guaraní). Cabe destacar que entre los integrantes de la Asesoría técnico-pedagógica figura Milda Rivarola.

En el manual Historia y Geografía 8 de la editorial Don Bosco el tema de la Guerra de la Triple Alianza queda circunscrito a la Unidad 3, en el capítulo titulado "La Epopeya de los Siglos" que ocupa un total de 21 páginas. De forma complementaria, la Unidad 4 "Ñamopu'ã jey Paraguái" (en lengua guaraní: Levantemos de nuevo al Paraguay), escrita de forma íntegra en lengua guaraní, dedica su primer apartado al período de postguerra bajo el título "Paraguái Pyahu".

En rigor, no hay en el capítulo referencias al pasado como la "edad de oro". Pero el párrafo inicial del capítulo sí hace mención a las características del Paraguay antes del inicio del conflicto bélico: “Los adelantos técnicos en materia industrial, el auge comercial y el desarrollo sociocultural quedaron truncados por la guerra que sostuvo el Paraguay contra la Argentina, el Brasil y el Uruguay durante un lustro", y agrega: "Esta contienda de gran magnitud, nunca antes sufrida por los pueblos hispanoamericanos, se conoce como La Epopeya de los Siglos" (Monte, Reyes y Dávalos, 2000: p. 271). En la redacción de antecedentes y causas se dedica espacio a las relaciones con los tres países que conforman la alianza contra el Paraguay, pero se dedica una mayor atención a explicar las tensiones con Argentina. En este manual, no se menciona a Inglaterra en el apartado de causas y tampoco se hacen referencias a una posible responsabilidad del gobierno paraguayo en el inicio de las hostilidades.

El manual se explaya en la explicación sobre la Doctrina del equilibrio, el Congreso Extraordinario reunido el 18 de marzo de 1865 y el Tratado Secreto de la Triple Alianza, que cuenta con un subapartado propio que recalca la alianza ofensiva y defensiva de los tres estados. El libro contiene elementos patrióticos a lo largo de la narración de la guerra como así también numerosas referencias al heroísmo del pueblo paraguayo y se aprecia una centralidad de la figura del mariscal López. En el relato del final de la guerra se menciona la célebre frase atribuida 
a López "muero con mi Patria". La siguiente frase también da cuenta de lo planteado aquí: “La batalla de Cerro Corá, el holocausto final del infortunio paraguayo, en donde López reparó sus errores y desaciertos, fue acogida con festejos en Asunción y en los campamentos aliados. Un gran número de prisioneros paraguayos, lloró la triste muerte de su mariscal" (p. 285).

El manual escolar Estudios Sociales 8, editado en 2002 por la editorial Santillana, es una obra colectiva creada por el Departamento Editorial de Santillana Paraguay, bajo la dirección de María José Peralta Heisecke. Los autores del manual son: Luciana De Loof Sosa, Ivonne Petersen, Patricia Piccolini y Armando Rivarola. La presencia de la editorial Santillana en Paraguay se remonta al año 1997, es una de las editoriales que se instala en el mercado de la producción de libros de texto escolares con el fuerte impulso de las reformas educativas implementadas en el país a comienzos de la década de 1990. El manual de la editorial Santillana está pensado para formar parte de los materiales didácticos de las instituciones privadas ubicadas sobre todo en la capital Asunción y las ciudades que conforman el área metropolitana que son las zonas geográficas más pobladas del país.

Este manual no dedica un capítulo específico al contenido de la Guerra de la Triple Alianza (como sí tienen, por ejemplo, el contenido de la Independencia o el Gobierno del Doctor Francia). El contenido se distribuye en dos capítulos con un total de 6 páginas. Los antecedentes y causas de la guerra ocupan apenas dos párrafos, vale la pena decir que el relato de la guerra que contiene este manual es radicalmente diferente al de los otros manuales analizados, resulta evidente que los autores se alejan del discurso hegemónico nacionalista.

Sobre los antecedentes y causas, Ilama la atención es que en primer lugar y como causa principal del relato se mencione la responsabilidad de Paraguay en la guerra: "Algunos historiadores afirman que la causa principal del estallido de la guerra fue la visión equivocada de Francisco Solano López sobre los acontecimientos en el Río de la Plata" (De Loof Sosa et 
al., 2002: p. 148). En segundo lugar, y siguiendo el orden de la narración, se alude a otra serie de causas, formuladas a modo de cita, pero sin aportan mayores detalles, tampoco se amplía la información en otros apartados. Ahora bien, si la causa principal hacía referencia a la responsabilidad del presidente López, las otras causas mencionadas ponen el foco en la Argentina y el Imperio del Brasil: “En cambio, otros estudiosos ya más contemporáneos buscan una explicación de mayor de complejidad, más allá del personaje del mariscal López", y se agrega: "Los problemas de límites fronterizos, la libre navegación de los ríos, el equilibrio de poder entre Brasil y Argentina y la formación de Argentina como nación son algunos de los aspectos que se tienen en cuenta al tratar de comprender las causas de la Guerra contra la Triple Alianza. (p. 148).

El manual Historia y Geografía Octavo Grado Educación Escolar Básica, de las autoras Nancy G. Zacarías de Funes y Alicia E. Zacarías de Ruiz Díaz, está editado por Editora Litocolor S.R.L. y está elaborado según el programa de estudio del área del año 2010. No lleva fecha de edición ni de impresión, pero de acuerdo con la actualización que presenta la bibliografía del manual, se considera como año de edición 2012. Como resultado de las averiguaciones realizadas, se puede decir que este manual circula sobre todo en escuelas públicas y escuelas privadas cuya matrícula escolar está conformada por estudiantes de poder adquisitivo medio y medio-bajo.

El manual de la editorial Litocolor dedica la Unidad 5 y parte de la Unidad 6 al contenido de la guerra en un total de 30 páginas aproximadamente. Si bien el análisis de este manual escolar se centra en la Unidad 5 y parte de la Unidad 6, resulta provechoso atender al último párrafo de la Unidad 4, referente al gobierno de Carlos Antonio López, porque aporta datos importantes acerca del discurso que presenta este manual escolar en relación con la construcción de un pasado heroico y glorioso truncado por la guerra: "Con sus apologistas y detractores, con sus críticos y partidarios, la era de los López hasta hoy constituye el momento dorado 
de la historia paraguaya con don Carlos Antonio [...]" (Zacarías y Zacarías, 2012: p. 74). Aunque no se mencione, la cita corresponde a Nidia R. Areces y Beatriz González de Bosio, incluida en el libro publicado en 2010 por las autoras El Paraguay durante los gobiernos de Francia y de los López, que forma parte de la Colección La Gran Historia del Paraguay. La narración de las causas menciona las tensiones con los países del Plata, la Doctrina del Equilibrio y el Tratado Secreto de la Triple Alianza. Es interesante mencionar que en los antecedentes y causas se encuentran algunos párrafos "calcados" de los manuales escolares de Irmina C. de Lezcano y de Luis G. Benítez, autores a los que no se referencia de forma explícita.

El manual escolar Historia y Geografía 8. Octavo Grado, de la editorial Atlas, está editado en el año 2016 y cuenta con una única autora, la profesora Águeda Crimi de Villasboa. Se emplea sobre todo en instituciones educativas privadas con estudiantes de nivel adquisitivo medio alto y alto. La editorial Atlas S. A. es una empresa del Grupo Editorial Atlas que se dedica a la producción de materiales en diversos soportes. Inicia sus actividades en el año 2000, siendo una empresa pionera en la elaboración de contenidos y materiales destinados a la práctica docente.

En el caso de este manual escolar, el contenido de la Guerra contra la Triple Alianza ocupa un total de 29 páginas del manual escolar y está distribuido en 2 unidades del libro. En el relato de la guerra que ofrece este manual escolar se observan alusiones al pasado próspero del Paraguay antes del inicio de la guerra: “El período de paz y progreso muy pronto iba a ser interrumpido por una de las guerras más cruentas de nuestra historia" (Crimi, 2016: p. 102). En las causas, el manual otorga suficiente visibilidad a los cuatro países contendientes, Paraguay, Argentina, Brasil y Uruguay. La balanza de la responsabilidad en el inicio de las hostilidades se inclina sobre todo hacia el Imperio del Brasil y la Argentina. No hay menciones acerca de la responsabilidad/ participación de Inglaterra en las causas de la guerra, tampoco hay reflexiones críticas acerca de una posible responsabilidad del 
gobierno paraguayo en el inicio del conflicto. Por otra parte, el tema del Tratado Secreto de la Triple Alianza cuenta con su propio apartado y el contenido del mismo resulta similar al encontrado en la mayoría de los manuales escolares.

\subsection{Construcción de la identidad nacional: "nosotros" y los "otros"}

En cuanto a los discursos que incorporan la mirada del otro, en el manual Estudios Sociales 2 de Lezcano desde el comienzo del relato queda claramente contrapuesta la situación y características del Paraguay en los años previos a la guerra y la situación de los tres países aliados. En el apartado citado, se afirma: “El país se hallaba vinculado con las naciones más adelantadas de Europa" (Lezcano, s/f.: 244). Como contrapunto, se dice de los países aliados: "En los demás países vecinos, el Brasil, el Uruguay y la Argentina existía una anarquía política, que ya llevaba perjudicando a dichos países en sus relaciones internas y externas" (p. 244). Finalmente es posible observar en el siguiente párrafo los rastros de un discurso que presenta al Paraguay como una nación avasallada y una identidad quebrada como consecuencia de la guerra: "Su población, cons- tituida por 1.300 .000 habitantes con que contaba el país al comienzo de la contienda, había quedado reducida a sólo 200.000, en su mayoría ancianos, niños y mujeres", y continúa: “El resto había quedado en los campos de batalla. De una nación floreciente, en la época de los López, sólo quedaban ruinas, desolación y luto" (p. 249).

El manual Paraguay Ñane Retã en general no introduce adjetivos o valoraciones negativas o recriminatorias hacia los países integrantes de la triple alianza, aspecto que sí se detecta en otros libros de texto. Resulta interesante que para hacer referencia a Argentina, Brasil o Uruguay el texto casi siempre utilice las mismas fórmulas: "tropas aliadas" o "los aliados", a diferencia de otros manuales escolares que de forma explícita emplean fórmulas como "los argentinos" y "los brasileños" de manera 
recurrente. Un repaso muy breve por el contenido muestra que al imperio del Brasil se lo nombra como "la amenaza brasileña", en relación con Argentina se recalca la negativa del país vecino al pedido de Francisco Solano López en relación con las actuaciones en Uruguay y la negativa al paso de tropas paraguayas por territorio argentino al comienzo del conflicto. El relato de las consecuencias de la guerra guarda similitud con otras narrativas encontradas, pero de nuevo este manual introduce una novedad con la inclusión del apartado “El problema de la tierra", que aborda la venta de tierras por parte del estado después de la guerra.

En el manual de la editorial Don Bosco la construcción de la imagen del "otro" aparece de forma muy temprana en el capítulo de la guerra, de forma específica en los subapartados "las cuestiones internaciones" y "Modelos de Estados diferentes". La imagen que allí se presenta de la Argentina y el Imperio del Brasil, gira en torno a las divergencias históricas de estos dos países y el Paraguay. Vale la pena decir que esta visión del otro, generada al comienzo del capítulo, es la que servirá como matriz orientadora del resto de la narración. Es decir, que cada vez que en los acontecimientos intervengan argentinos y brasileños prevalecerá o emergerá el carácter hostil de Argentina y Brasil hacia Paraguay. Evidentemente, hay algunas excepciones, sobre todo respecto de la imagen de Argentina, a la que se imprimirá algún matiz atenuante.

En el caso del manual escolar de la editorial Santillana en muchas ocasiones la narración emplea la fórmula "los aliados" cuando se refiere a las acciones llevadas a cabo por Argentina o Brasil, de manera indistinta. A diferencia de lo encontrado en la mayoría de los manuales, no siempre la imagen que se presenta del "otro" resulta hostil o negativa. Es decir, a lo largo de la narración se expresa de forma clara el antagonismo existente entre los países contendientes, Paraguay contra Argentina, Brasil y Uruguay, pero las alusiones explícitas al carácter hostil de los países de la Triple Alianza hacia el Paraguay no abundan.

En la mayoría de los manuales escolares analizados la construcción de un "nosotros", 
en tanto que nación paraguaya con identidad diferenciada, se cristaliza mediante dos procedimientos o momentos: durante los años de la contienda bélica donde el relato épico centrado en las hazañas e infortunios de los paraguayos recurre a lo emotivo, y durante el período de postguerra y reconstrucción de la nación donde el relato gira en torno a los encomiables esfuerzos de la población sobreviviente por salir adelante. En el manual escolar de Santillana esto no ocurre así. Episodios como el saqueo de Asunción, que en otros manuales tienen su propio apartado, están prácticamente ausentes en este relato escolar.

El manual se explaya especialmente en el contenido dedicado a la postguerra, donde no se habla de reconstrucción sino de "reorganización nacional", con las implicancias que tiene este concepto. Llama la atención la frase que se emplea en el relato para referirse a la población sobreviviente de la guerra, o al menos esta parece la intención: "la nación que se había ido gestando en su interior". Como novedad se menciona a los legionarios, $\mathrm{y}$ en los siguientes términos:

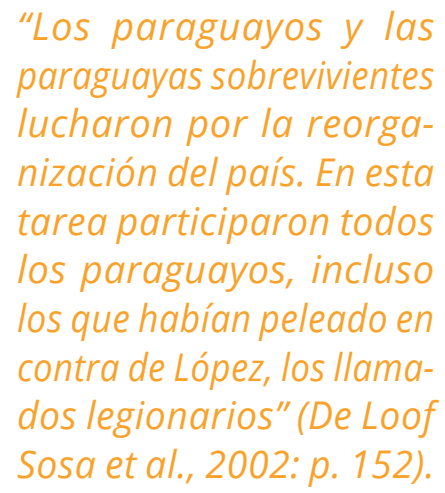

En el manual de la editorial Litocolor, la mirada del "otro" está centrada sobre todo en la Argentina y el Imperio del Brasil, en general se evidencia un discurso que construye de forma negativa la "otredad". Un momento interesante de la narración, que aparece casi sin excepción en todos los manuales analizados, es el apartado que trata de la entrevista de Yatayty Corá, en el que el Paraguay está representado en la figura de López y la Argentina está representada por el general Mitre. Se puede apreciar aquí la actitud amable o abierta del presidente López y la actitud por demás hostil de Mitre, que se extiende de alguna manera a la Argentina y los aliados. En cuanto a la construcción de un "nosotros" el manual destaca en todo momento las cualidades del pueblo paraguayo y el 
valor y arrojo del ejército, y en los apartados que hablan de la postguerra se construye una imagen de un Paraguay asolado por la guerra, cuya identidad nacional se ve amenazada por la inquina de los países vecinos.

El manual de la editorial Atlas proyecta una imagen de Argentina y el Imperio del Brasil en general hostil, en especial en las referencias encontradas hacia el Brasil. Especial importancia en el texto tiene el tema del saqueo de la ciudad de Asunción, que se menciona en más de un apartado y se detiene en la explicación de las condenables acciones por parte de los "invasores". Resulta muy interesante el contraste que suponen las descripciones llevadas a cabo del país antes del inicio del conflicto y la imagen que se presenta del país en los años de la postguerra, donde se erige una identidad nacional indefectiblemente ligada a la tragedia de la guerra y siempre en contraste con los países de la triple alianza.

Por otra parte, se incluyen numerosas referencias a la guerra como epopeya nacional. Hay que hacer notar también el empleo del plural mayestático, "nuestro ejército", para referirse al ejército o tropas paraguayas. También hay que decir que en este manual escolar el relato de las campañas y batallas ocupa un espacio importante en el conjunto, extendiéndose en 8 de las 29 páginas dedicadas al tema de la guerra. La figura de Francisco Solano López ocupa un lugar importante a lo largo de la narración y hay referencias al heroísmo y arrojo de los paraguayos. En el relato de las campañas y batallas de la guerra, destaca de forma particular la figura del general José Eduvigis Díaz.

El tema de la batalla de Acosta Ñu es ampliado en uno de los cuadros que acompañan al texto central bajo el título "¡Más info!". Aunque no se indica la fuente del extracto presentado, se trata de un fragmento muy conocido (y citado) del libro Genocidio Americano. La Guerra del Paraguay, del autor Chiavenato. Justamente, también aparece en el manual escolar de la editorial Litocolor, aunque en este caso se incluye como parte del texto central obviando la cita de la fuente original. Hay que decir que en 
el manual escolar de la editorial Atlas también se reproducen textos del historiador Julio César Cháves, correctamente citados, en relación con el episodio de
Cerro Corá. Igual que ocurría con el manual de Santillana, también aquí se introduce la célebre frase: "iMuero por mi patria con la espada en manos"!

\section{Conclusiones}

El recorrido propuesto aquí permite componer un panorama acerca de la representación de la Guerra de la Triple Alianza en los manuales escolares empleados desde 1989, momento en que se inicia el proceso de transición, hasta la actualidad. Si bien el estudio presentado forma parte de un trabajo de investigación más amplio, los manuales aquí analizados reflejan los diferentes discursos escolares de la guerra que fueron circulando por los espacios escolares durante las tres últimas décadas. En este sentido, es importante señalar que el estudio de los relatos escolares sobre la guerra a partir de una mirada histórica no supone una sucesión dialéctica de los mismos en el tiempo, muy por el contrario, estos relatos se entremezclan, pactan alianzas estratégicas, se alternan, se contraponen o conviven de forma conflictiva en la escuela actual.
En primer lugar, una mirada en perspectiva o diacrónica sobre los relatos escolares de la guerra en los manuales muestra que el discurso nacionalista heroico configurado en las primeras décadas del siglo XX y consolidado en el régimen de Stroessner ha experimentado variaciones desde el inicio de la transición. El manual escolar de la Estudios Sociales 2 (s/f) de la editorial Talleres Gráficos Comuneros reproduce el relato oficial de la historia presente en las aulas durante la etapa de la dictadura, es interesante constatar que la serie de manuales de Ciencias Sociales de esta editorial circuló en las escuelas tanto en la etapa de la dictadura como durante la primera década de la transición.

El manual escolar Paraguay Ñane Retã (1998) de la editorial En Alianza representa un punto de quiebre en el continuismo del relato escolar nacionalista. 
Es destacable el esfuerzo de los autores por presentar una narración renovada de la Guerra de la Triple Alianza donde además de los habituales aspectos bélicos y políticos, ahora se suman los aspectos sociales, culturales y económicos del conflicto, muchos de los cuales se abordan desde un enfoque crítico. En cierta medida, puede afirmarse que el abordaje del contenido de la guerra por parte de este manual tiene en cuenta las recomendaciones del documento "Propuestas de la Academia Paraguaya de la Historia para la reforma de programas y textos escolares de la materia" del año 1996. Aunque no forma parte del análisis aquí expuesto, es importante señalar que el manual escolar Historia y Geografía 8 editado en 2010 por la editorial En Alianza, cuya única autora de los contenidos del área de historia es Milda Rivarola, desarrolla el enfoque crítico que puede apreciarse en el manual escolar de 1998.

Con la introducción de la Reforma Educativa en el Tercer Ciclo de la Educación Escolar Básica en el año 1998 los antiguos y tan conocidos manuales como los de Irmina
C. de Lezcano y Luis G. Benítez comienzan a perder presencia como material didáctico en la enseñanza de la historia. En este contexto de reformas aparece el manual Historia y Geografía 8 Grado (2000) de la editorial Don Bosco que parece situarse en el umbral de los nuevos cambios, porque a diferencia del manual de la editorial En Alianza se sitúa en una posición intermedia en el que el relato de la guerra introduce aspectos y enfoques novedosos sin abandonar completamente algunos enunciados del discurso nacionalista. Hay que recordar que este manual es el resultado de una licitación realizada por el Ministerio de Educación y Cultura, de manera que puede decirse que representa el discurso oficial de la época.

Con la expansión del mercado editorial aparece el manual Historia y Geografía 8 de SantiIlana (2002) cuyo relato escolar se aleja de forma drástica del discurso nacionalista. Recogiendo lo más importante, la propuesta de esta editorial intenta insertar el complejo acontecimiento de la guerra en el nuevo escenario latinoamericano marcado por los procesos de globalización y 
por los esfuerzos gubernamentales orientados al refuerzo de integración de los países de la región. Todo ello, a riesgo de que el relato omita sucesos clave del desarrollo de la guerra y alcance, por momentos, cierta simplicidad y superficialidad.

En cuanto al manual Historia y Geografía 8 Grado (2012) de la editorial Litocolory el manual Historia y Geografía 8 Grado (2016) de la editorial Atlas presentan en general un relato de la guerra con características épicas. Sin embargo, es necesario introducir matices. El manual de la editorial Litocolor se presenta como la continuación de la línea discursiva de los manuales de historia de autores ya mencionados como Lezcano o Benítez, mientras que el relato de la guerra que contiene el manual de la editorial Atlas oscila entre el intento por reflejar los nuevos debates historiográficos y la reproducción de la visión patriótica y heroica del conflicto bélico.

En segundo lugar, desde una perspectiva actual, puede decirse que el discurso nacionalista donde la Guerra de la Triple Alianza se convierte en un acontecimiento articulador del pasado nacional continúa presente en la historia escolar. El recorrido a través de los manuales pone de manifiesto que existen diferentes formas de abordar los antecedentes y causas de la guerra, y asimismo ofrece una mayor comprensión de las diferentes miradas que construyen los manuales acerca de "nosotros/los otros" y como estas representaciones operan como un elemento configurador de la identidad nacional. Así, los manuales escolares que se alejan del discurso nacionalista rompen con la mirada simplista e intentan recoger diferentes enfoques que muestran la complejidad del proceso. Por otra parte, los manuales que en mayor o menor medida reproducen el relato heroico de la guerra, presentan una mirada hostil de los países vecinos; en estos relatos la construcción del sentimiento nacional se encuentra unida a la construcción negativa de la imagen del otro.

En resumen, en la actualidad existen al menos dos relatos escolares acerca de la guerra: uno más ligado a las emociones y a la formación de un ciudadano nacional y un relato concebido 
más allá de adscripciones identitarias nacionales y que, con mayor o menor éxito, intenta articular una narración que tiene en cuenta los conflictos inherentes de los procesos históricos. Las tensiones provocadas entre estos dos modelos que, como decíamos, unas veces se oponen y otras veces conviven de forma conflictiva, no hacen más que reflejar las tensiones presentes en los debates historiográficos y la emergencia de diferentes memorias de la guerra que acompañaron el inicio de la transición. A juzgar por los resultados encontrados, los cambios y las persistencias también se extienden al ámbito de los manuales escolares.

A mediados de la década de 1990 el pionero estudio de la Academia Paraguaya de la Historia sugería que la priorización otorgada en los manuales de historia a las dos guerras inter- nacionales debía ser relativizada dentro del conjunto de procesos socio-históricos relevantes del Paraguay. Y muchos estudios e investigaciones recientes sobre manuales escolares y la guerra concluyen en esta dirección. Casi tres décadas más tarde esta propuesta cobra especial interés y sentido. Porque es necesario considerar la guerra, su representación, en relación con un conjunto de procesos, es necesario quitar el carácter absoluto de la guerra y considerarla con un conjunto posible de significados, podría decirse que es necesario "desencializar", "desabsolutizar" la memoria de la guerra en la escuela. No para olvidar o borrar el pasado, sus héroes y las gestas, sino para ensayar una enseñanza de la historia que reconozca el conflicto, que incorpore pluralidad de aristas y que establezca una relación intrínseca y combativa con el presente. 


\section{Referencias bibliográficas:}

Abente, D. 2010. “Después de la dictadura (1989-2008)". En Ignacio Telesca (coord.), Historia del Paraguay, Asunción: Taurus, pp. 295-316.

Alegre, C. 2016. “Historia y memoria en la escuela paraguaya. La imagen de las mujeres en el relato escolar de la Guerra de la Triple Alianza", Novapolis, No. 10, pp. 31-49.

Barreto, A. 2013. "Las mujeres. Guerra de la Triple Alianza”, Asunción, El Lector.

Brezzo, L. 2001. “El Paraguay y la Argentina en los textos escolares: una perspectiva bilateral de las representaciones del otro", Entrepasados. Revista de Historia, No. 20- 21, 163-194.

Brezzo, L. 2008. “En el mundo de Ariadna y Penélope: hilos, tejidos y urdimbre del nacimiento de la historia en el Paraguay [Consideraciones en torno a la polémica Cecilio Báez-Juan O'Leary]". En Ricardo Scavonne Yegros y Sebastián Scavonne Yegros (comps.), Cecilio Báez-Juan O'Leary: polémica sobre la historia del Paraguay, Asunción, Tiempo de Historia, pp. 11-63.

Brezzo, L. 2009. “El Paraguay en cinco momentos historiográficos: retos y perspectivas", En Juan Manuel Casal y Thomas Whigham (eds.), Paraguay, el nacionalismo y la guerra. Actas de las Primeras Jornadas Internacionales de Historia del Paraguay en la Universidad de Montevideo, Asunción, Universidad de Montevideo-Servilibro, pp. 61-78.

Brezzo, L. 2010. "La Historia de la Guerra del Paraguay: nuevos enfoques, otras voces, perspectivas recientes", Observatorio Americano, No. 2, pp. 14-18.

Capdevila, L. 2006. “Memoria de guerra”, Revista Estudios Paraguayos, Vol., 24, No. 1-2, pp. 189-209.

Capdevila, L. 2009. “El macizo de la Guerra de la Triple Alianza como substrato de la identidad paraguaya", Nuevo Mundo Mundos Nuevos. Recuperado de https://journals.openedition.org/nuevomundo/48902 (Consultado el 05 de agosto de 2020)

Carretero, M. 2007. Documentos de identidad: la construcción de la memoria histórica en un mundo global, Buenos Aires, Paidós.

Consejo Nacional de Educación y Cultura. 2002. Situación de la Educación en el Paraguay. Informe del Consejo Nacional de Educación y Cultura, Asunción, CONEC.

Cuesta, R. 2011. Historia con memoria y didáctica crítica, Con-Ciencia Social, No. 15, pp. 15- 30. 
D'Alessandro, S. 2014. "Las representaciones del pasado reciente en los textos escolares de Historia en Paraguay", Discurso \& Sociedad, Vol. 8 No. 1, pp. 37-56.

Elías, R. 2014. “La Reforma Educativa paraguaya: base conceptual, políticas, planes y programas", Paraguay a Debate, Serie Notas de Política No. 17, pp. 1-17.

Elías, R. y Segovia, E. 2011. "La educación en tiempos de Stroessner". En Adriana Pesoa Nardi (ed.), La educación en el Paraguay independiente, Tomo I, Construyendo juntos la Nueva Escuela Pública Paraguaya, Asunción, Ministerio de Educación y Cultura, pp. 173-206.

Historia Paraguaya. Anuario de la Academia Paraguaya de la Historia. 1996. "Propuestas de la Academia Paraguaya de la Historia para la reforma de programas y textos escolares de la materia", Historia Paraguaya, Vol. XXXVI, pp. 255-293.

Jelin, E. y Lorenz, F. 2004. “Educación y memoria: entre el pasado, el deber y la posibilidad". En Elizabeth Jelin y Federico Lorenz (Comps.), Educación y memoria. La escuela elabora el pasado, Madrid, Siglo XXI, pp. 1-10.

Lambert, P. 2016. "Paraguayan National Identity". In Oxford Research Encyclopedia of Latin American History. Subject: History of Southern Spanish America, Cultural History, Social History, Oxford, Oxford University Press, pp. 1-23.

Nickson, A. 2010. “El régimen de Stroessner: 1954-1989”. En Ignacio Telesca (coord.), Historia del Paraguay, Asunción, Taurus, pp. 265-294.

Potthast, B. 2006. Algo más que heroínas. Varios roles y memorias femeninas de la Guerra de la Triple Alianza. Diálogos, Vol. 10, No. 1, pp. 89-104.

Rivarola, M. 1996. “Filosofías, pedagogías y percepción colectiva de la historia en el Paraguay", Historia Paraguaya. Anuario de la Academia Paraguaya de la Historia, Vol. 36, pp. 41-78.

Soler, L. 2010. “¿El mito de la isla? Acerca de la construcción del desconocimiento y la excepcionalidad de la historia política del Paraguay", Papeles de Trabajo, Vol. 4, No. 6, pp. 1-19.

Soler, L.; Elías, R. y Portillo, A. 2015. “El régimen stronista y su incidencia en la configuración y las prácticas escolares (1954-1970)". Cuadernos Chilenos de Historia de la Educación, Vol. 3, No. 4, pp. 10-33.

Telesca, I. 2015. “La guerra en la escuela. Textos de lectura y celebraciones escolares en el Paraguay de fines del siglo XIX e inicios del siglo XX". Folia Histórica del Nordeste, No. 24, pp. 131-150.

Velázquez, D. 2011. “Reparar el pasado”. En VV. AA., Paraguay: Ideas, Representaciones \& Imaginarios, Asunción, Secretaría Nacional de Cultura, 
pp. 175-187.

Velázquez, D. 2015. Relaciones entre autoritarismo y educación en Paraguay 1869-2012. Un análisis histórico. Primer volumen 1869-1930, Asunción, Servicio Paz y Justicia Paraguay.

Velázquez, D. 2018. "Autoritarismo, nacionalismo y militarismo en la educación paraguaya (1936-1989)", Diálogos, Vol. 22, No. 3, pp. 4-19.

\section{Fuentes}

Crimi, A. 2016. Historia y Geografía 8. Octavo Grado. Asunción, Atlas.

De Loof Sosa, L.; Petersen, I.; Piccolini, P. y Rivarola, A. 2002. Estudios Sociales 8. Asunción, Santillana.

Lezcano, I C. s/f. Estudios Sociales 2 Curso. Asunción, Comuneros.

Saccaggio, P. et al. 1998. Paraguay Ñane retã. Geografía, Historia, E. CívicaE. Sociales para el $3^{\circ}$. Ciclo de la Educación Escolar Básica. Asunción, En Alianza.

Monte de López Moreira, M.G.; Reyes Romero, A. y Dávalos, J. 2000. Historia y Geografía 8 Grado. Asunción, Don Bosco.

Rivarola, M., Villagra, R., Mussi, R. y Colombino, L. 2010. Historia \& Geografía 8. Asunción, En Alianza.

Zacarías de Funes, N. y Zacarías de Ruiz Díaz, A. 2012. Historia y Geografía Octavo Grado Educación Escolar Básica. Asunción, Editora Litocolor. 